\title{
Viral Coinfection among COVID-19 Patient Groups: An Update Systematic Review and Meta-Analysis
}

\author{
Pooneh Malekifar $\mathbb{D}^{1},{ }^{1}$ Reza Pakzad $\left(\mathbb{D},{ }^{2,3}\right.$ Ramin Shahbahrami $\mathbb{D}^{4}$ Milad Zandi ${ }^{4},{ }^{4,5}$ \\ Ali Jafarpour $\mathbb{D}^{6}{ }^{6}$ Sara Akhavan Rezayat $\mathbb{D}^{\circ},{ }^{7}$ Samaneh Akbarpour $\mathbb{D}^{\circ},{ }^{8}$

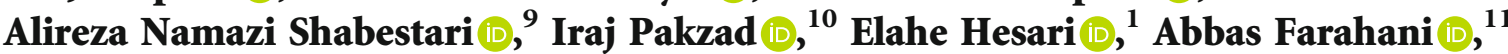 \\ and Saber Soltani $\mathbb{i D}^{4,5}$ \\ ${ }^{1}$ Department of Epidemiology, School of Public Health, Tehran University Medical Sciences, Tehran, Iran \\ ${ }^{2}$ Department of Epidemiology, Faculty of Health, Ilam University Medical Sciences, Ilam, Iran \\ ${ }^{3}$ Student Research Committee, Ilam University Medical Sciences, Ilam, Iran \\ ${ }^{4}$ Research Center for Clinical Virology, Tehran University of Medical Sciences, Tehran, Iran \\ ${ }^{5}$ Department of Virology, School of Public Health, Tehran University of Medical Sciences, Tehran, Iran \\ ${ }^{6}$ Gerash Amir-al-Momenin Medical and Educational Center, Gerash University of Medical Sciences, Gerash, Iran \\ ${ }^{7}$ Department of Management \& Health Economics, School of Public Health, Tehran University of Medical Sciences, Tehran, Iran \\ ${ }^{8}$ Occupational Sleep Research Center, Baharloo Hospital, Tehran University of Medical Sciences, Tehran, Iran \\ ${ }^{9}$ Department of Geriatric Medicine, School of Medicine, Tehran University of Medical Sciences, Tehran, Iran \\ ${ }^{10}$ Department of Microbiology, School of Medicine, Ilam University Medical Sciences, Ilam, Iran \\ ${ }^{11}$ Infectious and Tropical Diseases Research Center, Hormozgan Health Institute, Hormozgan University of Medical Sciences, \\ Bandar Abbas, Iran
}

Correspondence should be addressed to Abbas Farahani; abbasfarahani25@yahoo.com and Saber Soltani; sabersoltani71@gmail.com

Received 21 May 2021; Accepted 10 August 2021; Published 3 September 2021

Academic Editor: San-Ji Gao

Copyright (c) 2021 Pooneh Malekifar et al. This is an open access article distributed under the Creative Commons Attribution License, which permits unrestricted use, distribution, and reproduction in any medium, provided the original work is properly cited.

\footnotetext{
Background. Coinfections have a potential role in increased morbidity and mortality rates during pandemics. Our investigation is aimed at evaluating the viral coinfection prevalence in COVID-19 patients. Methods. We systematically searched scientific databases, including Medline, Scopus, WOS, and Embase, from December 1, 2019, to December 30, 2020. Preprint servers such as medRxiv were also scanned to find other related preprint papers. All types of studies evaluating the viral coinfection prevalence in COVID-19 patients were considered. We applied the random effects model to pool all of the related studies. Results. Thirty-three studies including 10484 patients were identified. The viral coinfection estimated pooled prevalence was 12.58\%; 95\% CI: 7.31 to 18.96 ). Blood viruses (pooled prevalence: $12.48 \%$; $95 \%$ CI: 8.57 to 16.93 ) had the most frequent viral coinfection, and respiratory viruses (pooled prevalence: $4.32 \%$; 95\% CI: 2.78 to 6.15 ) had less frequent viral coinfection. The herpesvirus pooled prevalence was $11.71 \%$ (95\% CI: 3.02 to 24.80). Also, the maximum and minimum of viral coinfection pooled prevalence were in AMRO and EMRO with 15.63\% (95\% CI: 3.78 to 33.31) and 7.05\% (95\% CI: 3.84 to 11.07), respectively. Conclusion. The lowest rate of coinfection belonged to respiratory viruses. Blood-borne viruses had the highest coinfection rate. Our results provide important data about the prevalence of blood-borne viruses among COVID-19 patients which can be critical when it comes to their treatment procedure.
} 


\section{Introduction}

The novel coronavirus outbreak started in Wuhan, China, and suddenly turned into one of the worst pandemics that have ever been experienced [1]. As of July 30, over 197 million infected persons and more than 4 million deaths were recorded [2]. Using different limitation methods to stop SARS-CoV-2 transmission, including national lockdown, was almost useless [3].

There are no practical clinical guidelines to manage the viral coinfection in COVID-19 patients, and their treatment takes place based on previous experience learned from other outbreaks including influenza and SARS [4].

SARS-CoV-2's extremely variable nature and insufficient information about host-pathogen interaction caused a great challenge to discover effective treatments against the virus [5]. Viral coinfection in COVID-19 patients may lead to more trouble in patient's recovery from the disease [6]. The interplay between different viruses and SARS-CoV-2 and their synergistic effect on disease clinical variation are under investigation [7].

The majority of respiratory viruses include respiratory syncytial virus human metapneumovirus and rhinovirus can cause coinfections; therefore, providing information about coinfection with these viruses in COVID-19 patients has a diagnostic value that can be helpful in the patients' outcome prediction [8]. It should be noted that providing data about coinfection with other respiratory viruses in COVID-19-confirmed cases could be crucial for patient antiviral therapy. Individuals with coinfections may show different reactions to treatment compared to those with only COVID-19 [9]. It is also considered that the synergy between COVID-19 and respiratory viruses could increase the disease progression. This matter is important especially among high-risk patients, including those with immunodeficiency and immunosuppression [10].

Several studies observed respiratory virus coinfection in COVID-19. About $5.8 \%$ of the confirmed COVID-19 cases in Wuhan were infected with other types of other respiratory viruses [11]. Another study in Northern California reported that the $20.7 \%$ positive COVID-19 specimens were infected with at least one more pathogens, and among them, respiratory viruses were the most coinfected agents [8]. Although based on different reports, the respiratory virus coinfection prevalence among COVID-19-infected people is surprisingly low. It is quite clear that enough information about the viral coinfection prevalence and types of viruses will help the clinician to run a better diagnosis and treat patients more precisely. This study was conducted to identify the viral coinfection prevalence among infected people with SARSCoV-2.

\section{Methods}

We performed this study according to PRISMA guidelines [12]. Our study design was registered in the International Prospective Register of Systematic Reviews with CRD42021240030 registration number. We identified all of the studies which had our desired keywords including
Coronavirus, COVID-19, SARS-CoV-2, SARS-CoV-2 infection, Polymicrobial Infection, Viral AND co-infections, Viral AND Secondary Infections, and Viral AND Mixed Infections.

2.1. Literature Search Method. An in-depth analysis of search engines, including Scopus, WOS, Medline, and Embase, was conducted to find relevant research papers on the viral coinfections among COVID-19-infected persons from December 1, 2019, to December 30, 2020. Preprint servers including medRxiv were also screened and data were retrieved. The authors selected keywords based on $\mathrm{MeSH}$ terms. Inclusion and exclusion criteria were defined using the PICO algorithms. We also designed our search strategy based on PICO algorithms. We considered both text words and $\mathrm{MeSH}$ terms to define our search keywords and their combinations were used to find the relevant articles.

A virologist identified the relevant articles. We send all of the related articles to Endnote X6. Afterward, we removed the duplicate articles. The remaining articles were reviewed in three steps. In the first step, we reviewed the title of the article and then the abstract, and finally, the article full texts were evaluated. Two authors reviewed the articles independently using these steps. The opinion of the third author was used to address the issues of "RR," "SS," and interrater discrepancies. We used both blinding and task separation procedures during the selection of the studies. The inter-rater agreement was $88 \%$.

2.2. Inclusion and Exclusion Criteria. All of the related crosssectional, case series studies, and cohort, were reviewed. The viral coinfection prevalence among patients was unrestricted. We excluded case series and case reports with less than 5 sample sizes, editorial, commentaries, case-control, and randomized clinical trials.

2.3. Data Extraction. In addition to general information, such as the name of authors, country, year, area, design of the study, COVID-19 patients numbers or sample size or, gender and age, other information, such as viral coinfections numbers and types, were extracted from all studies. We also included the COVID-19 patients (cases with positive COVID-19), and in addition, we even considered one viral coinfection in our study.

2.4. Subgroup Definition. We classified the viruses based on their transmission and clinical features. We categorized the countries according to the latest WHO guideline which contains six regions: Africa (AFRO), Americas (AMRO), Eastern Mediterranean (EMRO), Europe (EURO), South-East Asia (SEARO), and Western Pacific (WPRO).

2.5. Quality Assessment. The Newcastle-Ottawa Scale was applied to evaluate the quality of selected studies [13]. Two authors reviewed the articles separately, and the total score of each of the articles was calculated. Then, all of the selected studies were categorized based on these levels: very good, good, satisfactory, and unsatisfactory [14]. 


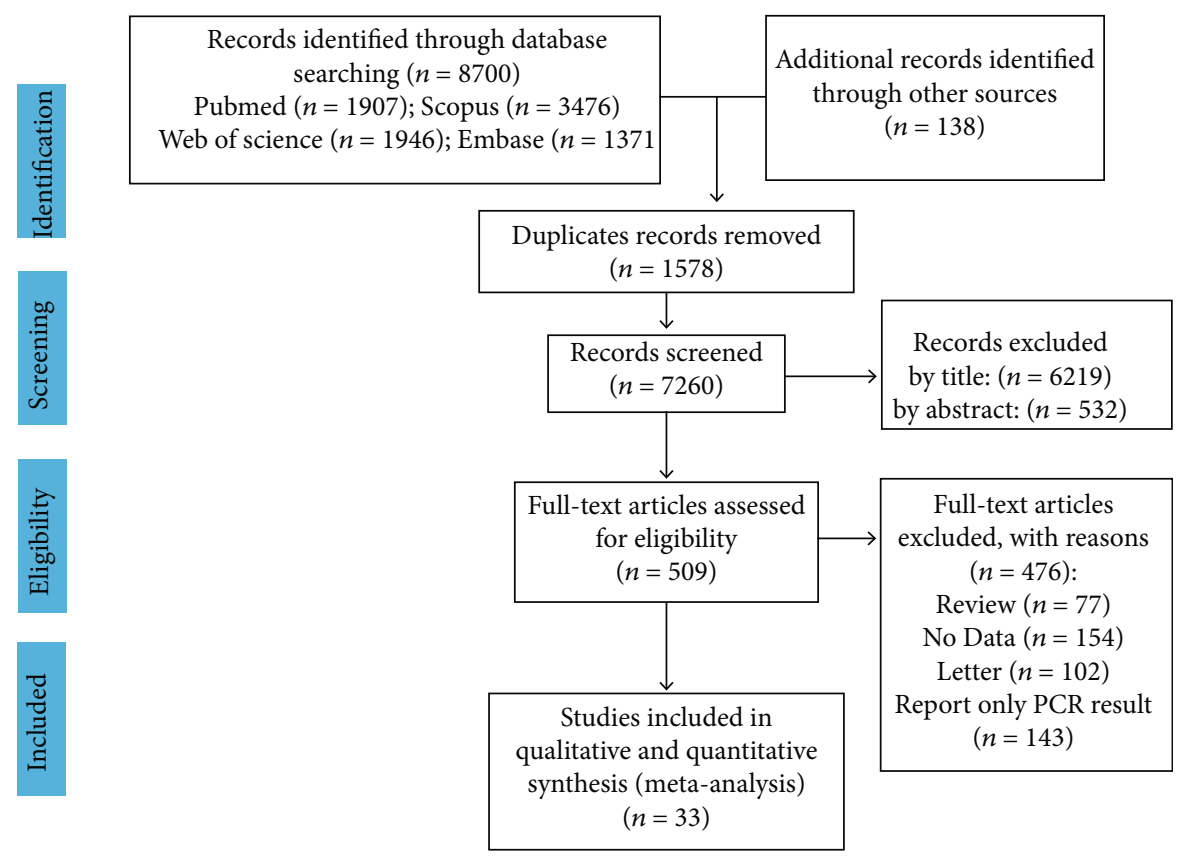

FIGURE 1: Study selection process PRISMA flow chart.

2.6. Statistical Analysis. We performed the analysis using Stata software 14.0. COVID-19 cases, viral coinfection prevalence, and types of viruses were extracted [14-18]. Cochran's $Q$ test was used to determine the heterogeneity. We also applied the $I^{2}$ index to quantify the heterogeneity. We considered the $I^{2}$ value above 0.7 as high heterogeneity based on Higgins classification. The "meta pop" command was applied to evaluate the pooled prevalence with a $95 \%$ confidence interval (CI), and we used a random effects plot to discover the pooled prevalence. We used the metaregression approach to evaluate the age, WHO region, and sample size effect on the study heterogeneity. Publication bias was checked by the "metabias" command. We adjusted the prevalence rate with the "metal trim" command to avoid any publication bias. A statistical significance of 0.05 was considered during the analysis.

\section{Result}

We found 8838 articles during the search process through scientific databases. Afterward, redundant papers were excluded and 7260 studies remained. We screened the articles in three independent steps. First, article titles were scanned: 6219 articles were excluded and 1041 studies remained. In the second step, the abstract was reviewed; we excluded 532 studies and in the final step; the full texts of the 509 remaining studies were scanned thoughtfully, and 476 studies were excluded. In the end, 33 studies [8, 19-49] with a total sample size of 10484 patients were analyzed. Figure 1 exhibits this selection procedure, and the study characteristics are available in Table 1 and supplement 1. The maximum number of articles was from the Western Pacific Region (16 studies), and the Eastern Mediterranean area had the minimum number of studies (2 studies). The publication date of all the articles was 2020. The highest and lowest patient age was recorded by Hughes (mean age $=69.5$ ) and $\mathrm{Wu}($ mean age $=6)$, respectively. It should be mentioned that $25(75.76 \%)$ of the articles were case series, $5(15.15 \%)$ cohorts, and $3(9.09 \%)$ cross-sectional.

3.1. Viral Coinfection Pooled Prevalence among COVID-19 Patients. We presented the viral coinfection prevalence in Table 1. Figure 2 shows the viral coinfection prevalence forest plot. Ebrahim in Saudi Arabia reported the lowest viral coinfection prevalence (prevalence: $0 \%$; 95\% CI: 0 to 3.66) [25], and the highest levels of coinfection prevalence (prevalence: 59.86 ; $95 \%$ CI: 51.47 to 67.85 ) [39] were recorded by Sharov in Russia. By performing a random effects model, the pooled estimated prevalence of viral coinfections was calculated at $12.58 \%$ (95\% CI: 7.31 to 18.96) (Figure 2). Our results indicated that from every 1000 COVID-19 patients, 73 to 190 individuals had viral coinfections.

3.2. Pooled Prevalence of Viral Coinfections Based on Different Subgroups. The viral coinfection pool prevalence is available in Figure 3 according to virus subtypes and the area of the study. The most frequent subtype of viral coinfections was blood viruses (pooled prevalence: $12.48 \%$; $95 \% \mathrm{CI}$ : 8.57 to 16.93 ), and the less frequent virus subtype was respiratory viruses (pooled prevalence: $4.32 \%$; 95\% CI: 2.78 to $6.15)$. The herpesvirus pooled prevalence was $11.71 \%(95 \%$ CI: 3.02 to 24.80). Also, the most and least pooled prevalence of viral coinfections was estimated in AMRO and EMRO with $15.63 \%$ (95\% CI: 3.78 to 33.31 ) and $7.05 \%$ (95\% CI: 3.84 to 11.07 ), respectively. The EURO and AMRO pooled prevalence is shown in Figure 3.

3.3. Heterogeneity and Meta-Regression. Heterogeneity exported data are available in Table 2. Cochran's heterogeneity $Q$ test exhibited that heterogeneity was significant in 
TABLE 1: Evaluated articles featured in the present meta-analysis.

\begin{tabular}{|c|c|c|c|c|c|c|}
\hline Author & Country & Design & Publication year & Mean age & Sample size & Viral coinfection prevalence $(95 \% \mathrm{CI})$ \\
\hline Zhu et al. [48] & China & Retrospective case series & 2020 & 51 & 257 & $31.52(25.89$ to 37.58$)$ \\
\hline Zheng et al. [47] & China & Retrospective case series & 2020 & 30.6 & 1001 & $0.40(0.11$ to 1.02$)$ \\
\hline Blasco et al. [19] & Spain & Retrospective case series & 2020 & 64 & 183 & $1.64(0.34$ to 4.72$)$ \\
\hline Contou et al. [22] & France & Retrospective case series & 2020 & 61 & 92 & 14.13 (7.74 to 22.95$)$ \\
\hline Chen et al. [20] & China & Retrospective case series & 2020 & 52.5 & 326 & 6.13 (3.79 to 9.32$)$ \\
\hline Chen et al. [21] & China & Retrospective case series & 2020 & 51 & 123 & $12.20(6.99$ to 19.32$)$ \\
\hline Luna et al. [23] & Brazil & Case series & 2020 & 48.49 & 115 & $11.30(6.16$ to 18.55$)$ \\
\hline Ding et al. [24] & China & Case series & 2020 & 50.2 & 115 & 4.35 (1.43 to 9.85$)$ \\
\hline Ebrahim [25] & Saudi Arabia & Case series & 2020 & 44 & 99 & $0.00(0.00$ to 3.66$)$ \\
\hline Garcia-Vidal et al. [26] & Spain & Retrospective case series & 2020 & 62 & 989 & $0.61(0.22$ to 1.32$)$ \\
\hline Hashemi et al. [27] & Iran & Case series & 2020 & - & 105 & $21.9(14.42$ to 31.03$)$ \\
\hline Hazra et al. [28] & Chicago & Cross-sectional & 2020 & - & 459 & $3.70(2.17$ to 5.86$)$ \\
\hline Hughes et al. [29] & UK & Retrospective case series & 2020 & 69.5 & 836 & $0.00(0.00$ to 0.44$)$ \\
\hline Jiang et al. [30] & China & Case series & 2020 & $\leq 14$ & 161 & $0.40(0.11$ to 1.02$)$ \\
\hline Kim et al. [8] & California & Cross-sectional & 2020 & 46.9 & 116 & 21.55 (14.46 to 30.15$)$ \\
\hline Leuzinger et al. [31] & Switzerland & Prospective case series & 2020 & 49 & 825 & 12.97 (10.75 to 15.46$)$ \\
\hline Li et al. [49] & China & Case series & 2020 & 57 & 32 & 15.63 (5.28 to 32.79$)$ \\
\hline Lin et al. [32] & China & Retrospective case series & 2020 & $18-65$ & 92 & $6.52(2.43$ to 13.66$)$ \\
\hline Lin et al. [33] & China & Retrospective case series & 2020 & 45 & 133 & 12.78 (7.63 to 19.67$)$ \\
\hline Lv et al. [34] & China & Retrospective cohort & 2020 & 62 & 354 & $0.28(0.01$ to 1.56$)$ \\
\hline Ma et al. [35] & China & Case series & 2020 & 45.5 & 250 & $8.80(5.60$ to 13.02$)$ \\
\hline Ma et al. [36] & China & Cross-sectional & 2020 & 67 & 93 & 49.46 (38.93 to 60.03$)$ \\
\hline Massey et al. [67] & USA & Retrospective case series & 2020 & 62.3 & 790 & 34.18 (30.87 to 37.60$)$ \\
\hline Motta et al. [37] & Multiplace* & Cohort & 2020 & - & 69 & $1.45(0.04$ to 7.81$)$ \\
\hline Nowak et al. [38] & New York & Retrospective case series & 2020 & 60.2 & 408 & 20.34 (16.54 to 24.58$)$ \\
\hline Sharov et al. [39] & Russia & Retrospective case series & 2020 & - & 147 & 59.86 (51.47 to 67.85$)$ \\
\hline Teotonio et al. [40] & Brazil & Retrospective case series & 2020 & 44.55 & 112 & 38.39 (29.36 to 48.06$)$ \\
\hline Vaughn et al. [41] & Michigan & Cohort & 2020 & 64.7 & 1705 & $0.53(0.24$ to 1.00$)$ \\
\hline Weissberg et al. [42] & Switzerland & Retrospective cohort & 2020 & 49 & 11 & 9.09 (0.23 to 41.28$)$ \\
\hline Wu et al. [43] & China & Retrospective case series & 2020 & 6 & 74 & 13.51 (6.68 to 23.45$)$ \\
\hline Yu et al. [44] & China & Prospective cohort & 2020 & 57 & 67 & 10.45 (4.30 to 20.35$)$ \\
\hline Yue et al. [45] & China & Retrospective case series & 2020 & - & 307 & 49.84 (44.11 to 55.57 ) \\
\hline Zhang et al. [46] & China & Retrospective case series & 2020 & 64.76 & 38 & 15.79 (6.02 to 31.25$)$ \\
\hline
\end{tabular}

CI: confidence interval; *Belgium, Brazil, France, Italy, Russia, Singapore, Spain, and Switzerland.

the articles $(P<0.001)$. The $I^{2}$ index total viral coinfections and their different subtypes were up to $90 \%$. Metaregression results also identified that the age (coefficient: 2 $\times 10^{-4} ; P=0.708$ ), sample size (coefficient: $-1 \times 10^{-4} ; P=$ 0.152 ), and WHO region size (coefficient: $2.225 ; P=0.605$ ) were not effective on heterogeneity (Figures $4(\mathrm{a})$ and $4(\mathrm{~b})$ ).

3.4. Publication Bias. Egger's test showed that there wasn't considerable publication bias in our study.

\section{Discussion}

Our review revealed that most studies about viral coinfection among COVID-19 patients were conducted in the Western Pacific area. The Eastern Mediterranean region recorded the lowest number of studies. The lowest coinfection prevalence was in Saudi Arabia, and the highest prevalence has been reported in China. The highest rate of coinfection related to blood viruses was $12.48 \%$, and the lowest rate of coinfection belonged to respiratory viruses. The highest coinfection prevalence and the lowest coinfection prevalence were in WPRO and EMRO, respectively. Respiratory syncytial virus (RSV) is the main diagnosed respiratory virus among COVID-19-infected persons. A systematic review showed that RSV is a commonly isolated respiratory virus from different age groups and is the lower respiratory tract infection (LRTI) main causative agent in young children. Respiratory viruses are cross-species transmittable and exhibit close clinical symptoms to COVID-19, which turns them into a potential threat to people infected with COVID-19 [50, 51].

Respiratory viruses may remain infectious and continue to circulate and cause coinfection during new respiratory outbreaks; however, their coinfection with COVID-19 is estimated to be $0-3 \%$. However, recent studies suggested a higher coinfection incidence with other respiratory viruses [51, 52]. 


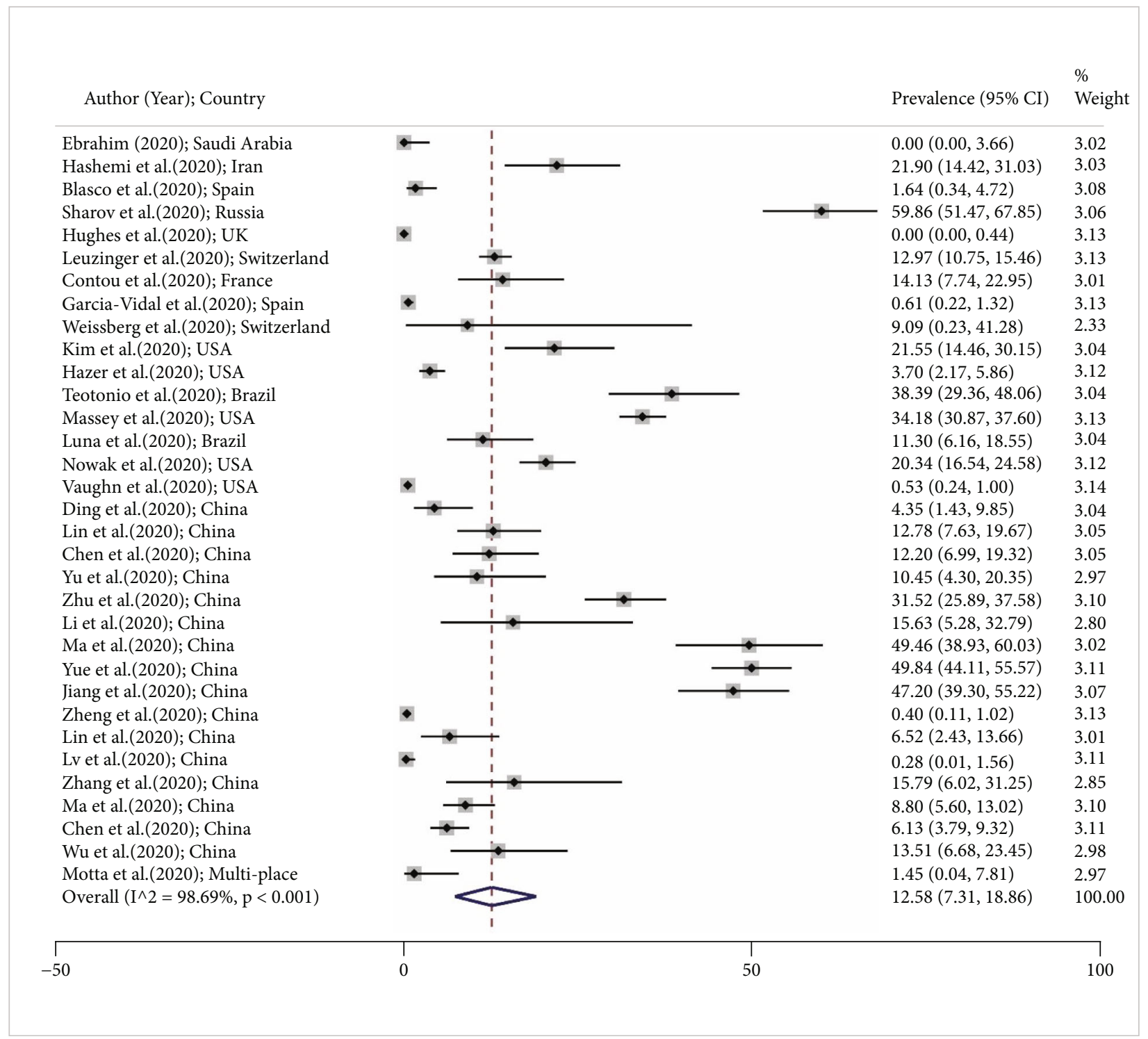

FIGURE 2: Forest plot shows prevalence of viral coinfections among COVID-19 patients according to the random effects approach. Every single article demonstrated by the first author (year) and country. Each line segment's midpoint exhibited the prevalence estimation, the line segment length presents $95 \%$ confidence interval (CI) in every study, and the diamond mark points out the pooled estimation.

It is difficult to accurately estimate the prevalence of SARS-CoV-2 coinfection with other types of respiratory viruses. Their similar nature and common clinical feature, as well as the lack of enough diagnostic equipment, are the major factors that limit us to distinguish them from each other. Therefore, it can be expected that the prevalence of these viruses among the patients is higher than those of the previous reports [53].

Another study showed that $11.6 \%$ of COVID-19 patients had coinfection. This study concluded that coinfection with respiratory viruses is common among COVID-19 patients [52].

Accurate estimation of the respiratory virus's coinfection prevalence rate among infected people with SARS-CoV-2 will lead to a better understanding of their role in the disease and also improve the diagnosis and course of treatment [54]. Our results indicated that the highest prevalence of coinfec- tion belonged to blood-borne viruses (BBVs). Our results are in contrast with other studies which reported RSV as the most common virus among COVID-19 patients $[35,52]$.

There are only a limited number of studies about SARSCoV-2 and HIV coinfection [55]; some people living with HIV (PLHIV) and especially males affected by ARVrelated complications could be more prone to severe COVID-19 disease [56]. The provided data about PLHIV showed that older patients may have poor morbidity and mortality condition with SARS-CoV2-HIV coinfection. A systematic review found that COVID-19-HIV patients had comorbidities with hypertension with $39.3 \%$ and $19.3 \%$ for obesity or hyperlipidemia, $18.0 \%$ had chronic obstructive pulmonary disease, and $17.2 \%$ of them had diabetes, and the majority of these patients were males over 50 years old $[57,58]$. 


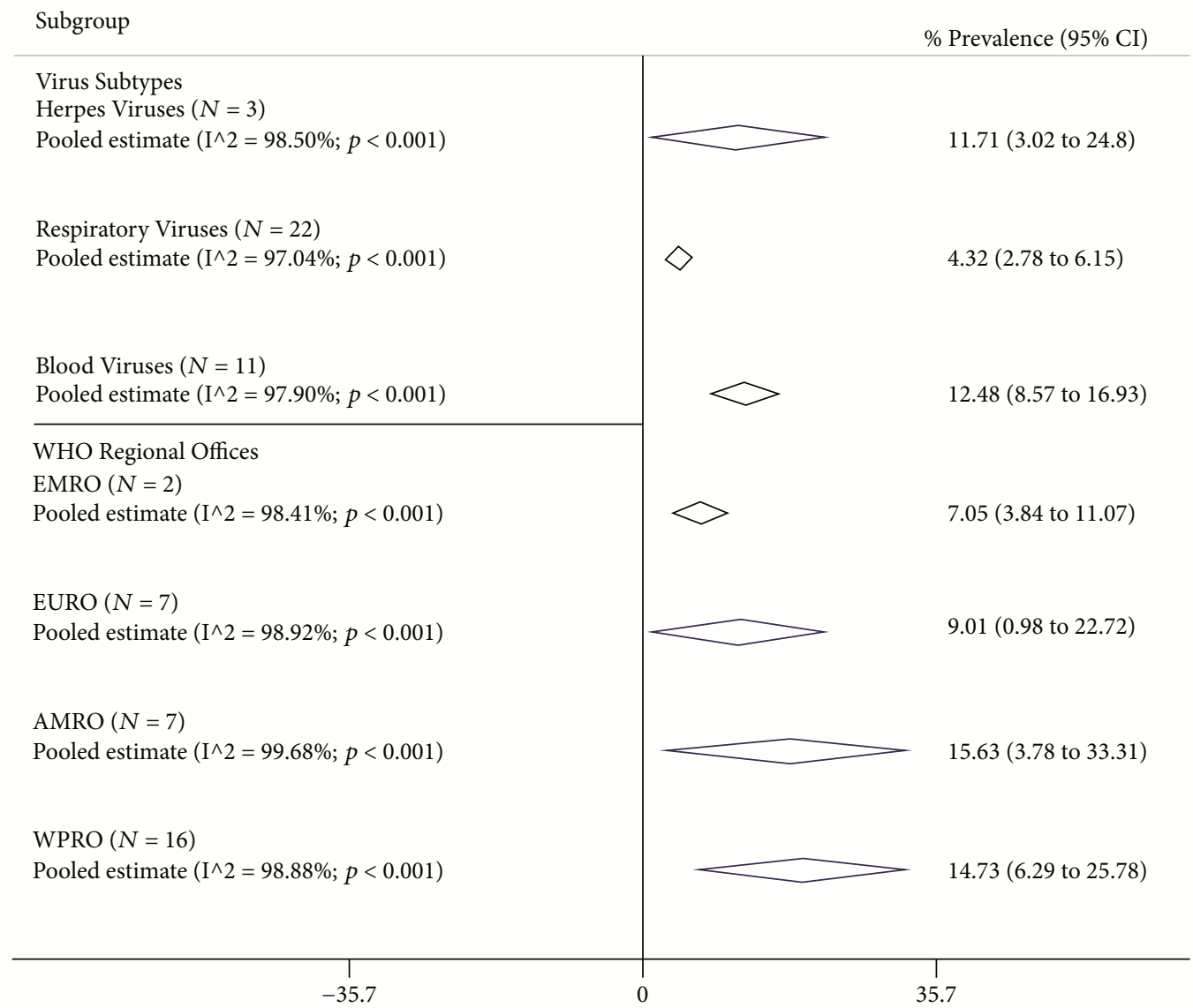

FIGURE 3: Pooled prevalence with 95\% confidence interval (CI) and heterogeneity indexes of viral coinfections among the COVID-19 patient based on the virus type and different region. The diamond mark exhibits the pooled prevalence and the diamond length shows $95 \%$ CI.

TABLE 2: The univariate meta-regression analysis on the determinant heterogeneity in viral coinfections among COVID-19 patient studies.

\begin{tabular}{lccc}
\hline Variables & Coefficient & $95 \%$ CI & $P$ value \\
\hline Age (year) & $2 \times 10^{-4}$ & $-4 \times 10^{-3}$ to $5 \times 10^{-3}$ & 0.897 \\
WHO region (score) & 2.225 & -6.317 to 11.404 & 0.598 \\
Sample size (number) & $-1 \times 10^{-4}$ & $-27 \times 10^{-5}$ to $2 \times 10^{-5}$ & 0.090 \\
\hline
\end{tabular}

CI: confidence interval; coding of WHO region: 1: EMRO; 2: EURO; 3: AMRO; 4: WPRO.

Coinfection between hepatitis viruses and SARS-CoV-2 is also quite controversial. SARS-CoV-2-HBV cases showed $4.7 \%$ and $15 \%$ mortality rates in cross-sectional and case report studies, respectively. SARS-CoV-2-HCV cases had an $8.3 \%$ mortality rate [59]. Regardless of infection with these two viruses, these patients had at least one comorbidity factor, including type 2 diabetes and hypertension. Liver enzyme abnormalities and acute hepatic injuries were observed in HBV and HCV patients, although it is yet unknown that the liver damage in these patients is related to COVID-19 or the hepatitis viruses and the interaction between these viruses and SARS-CoV-2 [60, 61]. Another study found that the liver damage prevalence among COVID-19 patients was $4 \%$ and caused by HBV. However, the study stated that there wasn't any relationship between COVID-19 coinfection in patients with chronic hepatitis and increased mortality rate.

In contrast to that, another research concluded that there are a considerable risk of mortality and morbidity between infected people with SARS-CoV-2 and HBV and HCV [59, 62]. Our result indicated that the highest rate of coinfection with COVID-19 belongs to blood-borne viruses. This result may happen because of a lack of enough research on respiratory coinfection with COVID-19 or low sample size in the studies on the subject. Another possible reason for this result could be the concurrent infection with the blood virus.

Immunocompromised patients are susceptible to viruses, especially herpes viruses, such as human HCMV and EBV. 


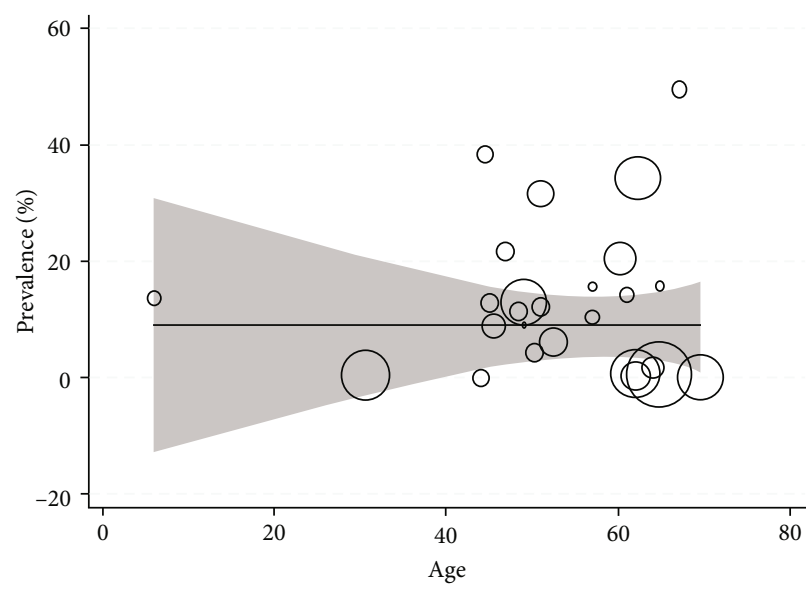

(a)

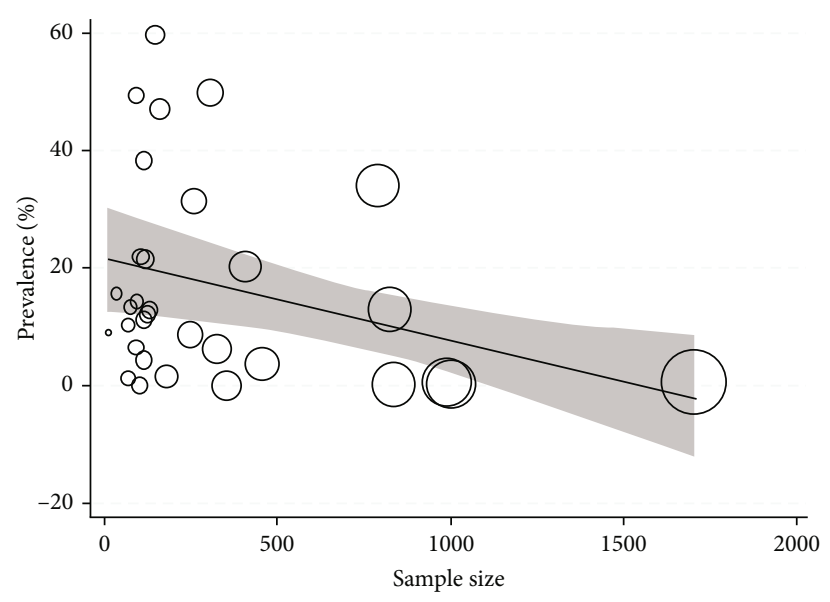

(b)

FIgURE 4: Association among (a) age prevalence and (b) sample size with viral coinfection prevalence by applying meta-regression. The circle size shows each study's precision. There is no considerable association with respect to the viral coinfection prevalence with age sample size.

This virus reactivation in intensive care unit (ICU) patients is associated with their morbidity and mortality [63]. EBV DNA was observed in $95.2 \%$ of the ICU patients and $83.6 \%$ of the SICU patients infected with COVID-19 [64]. EBV reactivation is notably related to more prolonged ICU length of stay in COVID-19 patients [65]. COVID-19 patients show a reduction in $\mathrm{NK}$ and $\mathrm{CD} 8+\mathrm{T}$ cells and the presence of EBV DNA. Also, a higher count of B cells in people with severe COVID-19 infection compared to patients with a mild form of the disease was observed [64]. The impact of an increase in EBV DNA on B-cell function is not pretty clear, but it can be postulated that there is a potential impact of the virus on COVID-19 severity. EBV has a distinguished role in COVID-19-infected cases compared to HCMV and HHV-6; among herpesviruses, only EBV reactivated in the patients. There wasn't a significant relationship between HHV- 6 and CMV reactivation and clinical outcomes in COVID-19 patients.

Reactivation of EBV occurs in the early phase after patients being admitted to the ICU. It is evident that critically ill patients are vulnerable to latent viruses, especially EBV reactivation, and due to their relevance with morbidity and mortality in immunocompromised individuals such as ICU and transplanted patients, more research is needed to investigate their association with COVID-19 and their impact on the different outcome of the COVID-19 infection [64, 65].

Infections with rhinovirus were also investigated during COVID-19 pandemics. Kim et al. [8] showed that rhinovirus infection prevalence was $6.9 \%$. The prevalence of nonpolio enterovirus activity decreased due to the COVID-19 pandemic and nonpharmaceutical interventions. For example, a lower incidence of NPEV was observed during the 20192020 season in Taiwan $[8,66]$. The prevalence of viral coinfection with COVID-19 may be affected by other viral outbreaks, such as influenza virus outbreaks and different anti-COVID measurements like nonpharmaceutical measurement and its effect on enterovirus activity in Taiwan.
There was high heterogeneity between the coinfection prevalence in different countries $\left(I^{2}=98 \%\right)$, which indicates that a large part of the calculated total variance in our study was because of the differences between these studies. Even combining the results of these researches with the random effects method was not helpful, and the metaregression method could not find the heterogeneity causative agents. Therefore, the mean age, sample size, and study location had no effect on heterogeneity among the studies. Also, there wasn't a similar meta-analysis study that evaluated different variable's effects on heterogeneity. We found only a few preliminary studies that evaluated the effects of different variables such as gender, length of stay, and hospitalization in the ICU $[49,55,56,58,60,67]$. Due to the low number of studies and their similar results, using metaregression methods was not applicable to investigate the variable impact.

There was also some limitation in our study: first, because there wasn't enough data, we could not perform the gender-specific estimation. This means that the number of studies reporting the gender prevalence was limited and we could not calculate robust gender pooled prevalence.

Second, we tend to calculate the pooled prevalence based on WHO territorial office data and perform spatial analysis in several geographical districts to distinguish the high-risk area for viral coinfection [68-76], but this estimation may be unreliable because of the small number of studies.

The current study's strengths were conducting a comprehensive search using various strategies such as searching online databases and preprint servers, manual searching to detect related articles, and estimating the pooled prevalence based on different subtypes of viruses and WHO regions.

\section{Conclusion}

Our study demonstrated that COVID-19 coinfections are prevalent among infected patients with blood-borne viruses (BBVs) such as HIV or HCV and surprisingly, the lowest rate of coinfection belonged to respiratory viruses. Due to 
the prevalence of influenza virus or RSV among societies, more studies need to be done to clarify the incidence rate of viral coinfection in COVID-19 patients. Another important aspect that should be investigated is their relationship with the morbidity and mortality of the patient. In a nutshell, further investigation about viral coinfection with SARS-CoV-2 is an urgent need.

\section{Data Availability}

All data can be obtained from corresponding authors Abbas Farahani and Saber Soltani or the co-first authors Pooneh Malekifar and Reza Pakzad.

\section{Conflicts of Interest}

The authors have no conflicts of interest to declare.

\section{Authors' Contributions}

The study concept and design were done by SS and AF; the analysis and interpretation of data were done by RP, PM, IP, and $\mathrm{EH}$; the drafting of the manuscript was done by RS, MZ, AJ, and SAR; the critical revision of the manuscript for important intellectual content was done by SS, RP, SA, AF, and ANS; the statistical analysis was done by RP, PM, and EH. Reza Pakzad and Pooneh Malekifar contributed equally to this work as co-first authors.

\section{Acknowledgments}

The present study is funded by Hormozgan University of Medical Sciences, Bandar Abbas, Iran (G. no. 4000280). We gratefully acknowledge the vice chancellor for the Research and Technology, Hormozgan University of Medical Sciences, Bandar Abbas, Iran. Also, we are sincerely thankful to our counselors in the Clinical Research Development Center of Shahid Mohammadi Hospital, Hormozgan University of Medical Sciences, Bandar Abbas, Iran. We gratefully acknowledge the Tehran University of Medical Sciences, Tehran, Iran.

\section{Supplementary Materials}

Supplement 1: type of virus coinfection in included studies. (Supplementary Materials)

\section{References}

[1] E. Blandenier, Z. Habibi, T. Kousi, P. Sestito, A. Flahault, and L. Rozanova, "Initial COVID-19 outbreak: an epidemiological and socioeconomic case review of Iran," International Journal of Environmental Research and Public Health, vol. 17, no. 24, p. 9593, 2020.

[2] Organization WH, Weekly epidemiological update-2 February 2021, World Health Organization, 2021.

[3] H. R. GÜNER, I. Hasanoğlu, and F. Aktaş, "COVID-19: prevention and control measures in community," Turkish Journal of Medical Sciences, vol. 50, no. SI-1, pp. 571-577, 2020.
[4] E. Latreille and W. L. Lee, "Interactions of influenza and SARS-CoV-2 with the lung endothelium: similarities, differences, and implications for therapy," Viruses, vol. 13, no. 2, p. 161, 2021.

[5] R. B. Martines, J. M. Ritter, E. Matkovic et al., "Pathology and pathogenesis of SARS-CoV-2 associated with fatal coronavirus disease, United States," Emerging Infectious Diseases, vol. 26, no. 9, p. 2005, 2020.

[6] E. Sharifipour, S. Shams, M. Esmkhani et al., "Evaluation of bacterial co-infections of the respiratory tract in COVID-19 patients admitted to ICU," BMC Infectious Diseases, vol. 20, no. 1, pp. 1-7, 2020.

[7] V. Singh, P. Upadhyay, J. Reddy, and J. Granger, "SARS-CoV2 respiratory co-infections: incidence of viral and bacterial copathogens," International Journal of Infectious Diseases, vol. 105, pp. 617-620, 2021.

[8] D. Kim, J. Quinn, B. Pinsky, N. H. Shah, and I. Brown, "Rates of co-infection between SARS-CoV-2 and other respiratory pathogens," Journal of the American Medical Association, vol. 323, no. 20, pp. 2085-2086, 2020.

[9] C.-C. Lai, C.-Y. Wang, and P.-R. Hsueh, "Co-Infections among Patients with COVID-19: The Need for Combination Therapy with Non-Anti-SARS-CoV-2 Agents?," Journal of Microbiology, Immunology and Infection, vol. 53, no. 4, pp. 505-512, 2020.

[10] Y. Gao, Y. Chen, M. Liu, S. Shi, and J. Tian, "Impacts of immunosuppression and immunodeficiency on COVID-19: a systematic review and meta-analysis," The Journal of Infection, vol. 81, no. 2, pp. e93-e95, 2020.

[11] M. Wang, Q. Wu, W. Xu et al., "Clinical diagnosis of 8274 samples with 2019-novel coronavirus in Wuhan," MedRxiv, 2020.

[12] D. Moher, A. Liberati, J. Tetzlaff, D. G. Altman, and Group P, "Preferred reporting items for systematic reviews and metaanalyses: the PRISMA statement," PLoS Medicine, vol. 6, no. 7, article e1000097, 2009.

[13] J. Peterson, V. Welch, M. Losos et al., "The Newcastle-Ottawa Scale (NOS) for assessing the quality of nonrandomised studies in meta-analyses," Ottawa: Ottawa Hospital Research Institute, vol. 2, no. 1, pp. 1-12, 2011.

[14] H. Hashemi, R. Pakzad, A. Yekta et al., "Global and regional prevalence of age-related cataract: a comprehensive systematic review and meta-analysis," Eye, vol. 34, no. 8, pp. 1357$1370,2020$.

[15] J. Hallajzadeh, M. Khoramdad, N. Izadi et al., "Metabolic syndrome and its components in premenopausal and postmenopausal women: a comprehensive systematic review and metaanalysis on observational studies," Menopause, vol. 25, no. 10, pp. 1155-1164, 2018.

[16] H. Hashemi, R. Pakzad, S. Heydarian et al., "Global and regional prevalence of strabismus: a comprehensive systematic review and meta-analysis," Strabismus, vol. 27, no. 2, pp. 5465, 2019.

[17] H. Hashemi, R. Pakzad, A. Yekta et al., "Global and regional estimates of prevalence of amblyopia: a systematic review and meta-analysis," Strabismus, vol. 26, no. 4, pp. 168-183, 2018.

[18] S. Soltani, A. Tabibzadeh, A. Zakeri et al., "COVID-19 associated central nervous system manifestations, mental and neurological symptoms: a systematic review and meta-analysis," Reviews in the Neurosciences, vol. 32, no. 3, pp. 351-361, 2021.

[19] M. L. Blasco, J. Buesa, J. Colomina et al., "Co-detection of respiratory pathogens in patients hospitalized with 
coronavirus viral disease-2019 pneumonia," Journal of Medical Virology, vol. 92, no. 10, pp. 1799-1801, 2020.

[20] L. Chen, S. Huang, J. Yang et al., "Clinical characteristics in patients with SARS-CoV-2/HBV Co-infection," Journal of Viral Hepatitis, vol. 27, no. 12, pp. 1504-1507, 2020.

[21] X. Chen, Q. Jiang, Z. Ma et al., "Clinical characteristics of hospitalized patients with SARS-CoV-2 and hepatitis B virus co-infection," Virologica Sinica, vol. 35, no. 6, pp. 842-845, 2020.

[22] D. Contou, A. Claudinon, O. Pajot et al., "Bacterial and viral co-infections in patients with severe SARS-CoV-2 pneumonia admitted to a French ICU," Annals of Intensive Care, vol. 10, no. 1, p. 119, 2020.

[23] L. K. de Souza Luna, A. H. Perosa, D. D. Conte et al., "Different patterns of Influenza A and B detected during early stages of COVID-19 in a university hospital in Sao Paulo, Brazil," The Journal of infection, vol. 81, no. 2, pp. e104-e105, 2020.

[24] Q. Ding, P. Lu, Y. Fan, Y. Xia, and M. Liu, "The clinical characteristics of pneumonia patients co-infected with 2019 novel coronavirus and influenza virus in Wuhan, China," Journal of Medical Virology, vol. 92, no. 9, pp. 1549-1555, 2020.

[25] S. H. Ebrahim, "Lack of MERS-CoV Co-infection among hospitalized COVID-19 patients in Saudi Arabia," Journal of epidemiology and global health, vol. 10, no. 3, p. 191, 2020.

[26] C. Garcia-Vidal, G. Sanjuan, E. Moreno-García et al., "Incidence of co-infections and superinfections in hospitalized patients with COVID-19: a retrospective cohort study," Clinical Microbiology and Infection, vol. 27, no. 1, pp. 83-88, 2021.

[27] S. A. Hashemi, S. Safamanesh, H. Ghasemzadeh-moghaddam, M. Ghafouri, and A. Azimian, "High prevalence of SARSCoV-2 and influenza A virus (H1N1) Co-infection in dead patients in Northeastern Iran," Journal of Medical Virology, vol. 93, no. 2, pp. 1008-1012, 2021.

[28] A. Hazra, M. Collison, J. Pisano, M. Kumar, C. Oehler, and J. P. Ridgway, "Co-infections with SARS-CoV-2 and other respiratory pathogens," Infection Control \& Hospital Epidemiology, vol. 41, no. 10, pp. 1228-1229, 2020.

[29] S. Hughes, O. Troise, H. Donaldson, N. Mughal, and L. S. Moore, "Bacterial and fungal co-infection among hospitalized patients with COVID-19: a retrospective cohort study in a UK secondary-care setting," Clinical Microbiology and Infection, vol. 26, no. 10, pp. 1395-1399, 2020.

[30] S. Jiang, P. Liu, G. Xiong et al., "Co-infection of SARS-CoV-2 and multiple respiratory pathogens in children," Clinical Chemistry and Laboratory Medicine (CCLM), vol. 58, no. 7, pp. 1160-1161, 2020.

[31] K. Leuzinger, T. Roloff, R. Gosert et al., "Epidemiology of severe acute respiratory syndrome coronavirus 2 emergence amidst community-acquired respiratory viruses," The Journal of Infectious Diseases, vol. 222, no. 8, pp. 1270-1279, 2020.

[32] D. Lin, L. Liu, M. Zhang et al., "Co-infections of SARS-CoV-2 with multiple common respiratory pathogens in infected patients," Science China Life Sciences, vol. 63, no. 4, pp. 606609, 2020.

[33] Y. Lin, J. Yuan, Q. Long et al., "Patients with SARS-CoV-2 and HBV Co-infection are at risk of greater liver injury," Genes \& Diseases, vol. 8, no. 4, pp. 484-492, 2020.

[34] Z. Lv, S. Cheng, J. Le et al., "Clinical characteristics and Coinfections of 354 hospitalized patients with COVID-19 in Wuhan, China: a retrospective cohort study," Microbes and Infection, vol. 22, no. 4, pp. 195-199, 2020.
[35] L. Ma, W. Wang, J. M. Le Grange et al., "Co-infection of SARSCoV-2 and other respiratory pathogens," Infection and Drug Resistance, vol. 13, p. 3045, 2020.

[36] S. Ma, X. Lai, Z. Chen, S. Tu, and K. Qin, "Clinical characteristics of critically ill patients co-infected with SARS-CoV-2 and the influenza virus in Wuhan, China," International Journal of Infectious Diseases, vol. 96, pp. 683-687, 2020.

[37] I. Motta, R. Centis, L. D’Ambrosio et al., “Tuberculosis, COVID-19 and migrants: preliminary analysis of deaths occurring in 69 patients from two cohorts," Pulmonology, vol. 26, no. 4, pp. 233-240, 2020.

[38] M. D. Nowak, E. M. Sordillo, M. R. Gitman, and A. E. Paniz Mondolfi, "Co-infection in SARS-CoV-2 infected patients: Where are influenza virus and rhinovirus/enterovirus?," Journal of Medical Virology, vol. 92, no. 10, pp. 1699-1700, 2020.

[39] K. S. Sharov, "SARS-CoV-2-related pneumonia cases in pneumonia picture in Russia in March-May 2020: secondary bacterial pneumonia and viral co-infections," Journal of Global Health, vol. 10, no. 2, article 020504, 2020.

[40] I. M. Teotônio, J. L. de Carvalho, L. C. Castro et al., "Clinical and biochemical parameters of COVID-19 patients with prior or active dengue fever," Acta Tropica, vol. 214, article 105782, 2021.

[41] V. M. Vaughn, T. N. Gandhi, L. A. Petty et al., "Empiric antibacterial therapy and community-onset bacterial co-infection in patients hospitalized with COVID-19: a multi-hospital cohort study," Clinical Infectious Diseases: An Official Publication of the Infectious Diseases Society of America, vol. 72, no. 10, pp. e533-e541, 2020.

[42] D. Weissberg, J. Böni, S. K. Rampini et al., "Does respiratory co-infection facilitate dispersal of SARS-CoV-2? investigation of a super-spreading event in an open-space office," Antimicrobial Resistance \& Infection Control, vol. 9, no. 1, pp. 1-8, 2020.

[43] Q. Wu, Y. Xing, L. Shi et al., "Co-infection and other clinical characteristics of COVID-19 in children," Pediatrics, vol. 146, no. 1, 2020.

[44] R. Yu, S. Tan, Y. Dan et al., "Effect of SARS-CoV-2 coinfection wasntapparent on the dynamics of chronic hepatitis B infection," Virology, vol. 553, pp. 131-134, 2021.

[45] H. Yue, M. Zhang, L. Xing et al., "The epidemiology and clinical characteristics of co-infection of SARS-CoV-2 and influenza viruses in patients during COVID-19 outbreak," Journal of Medical Virology, vol. 92, no. 11, pp. 2870-2873, 2020.

[46] H. Zhang, Y. Zhang, J. Wu et al., "Risks and features of secondary infections in severe and critical ill COVID-19 patients," Emerging Microbes \& Infections, vol. 9, no. 1, pp. 1958-1964, 2020.

[47] X. Zheng, H. Wang, Z. Su et al., "Co-infection of SARS-CoV-2 and influenza virus in early stage of the COVID-19 epidemic in Wuhan, China.," The Journal of Infection, vol. 81, no. 2, p. e128, 2020.

[48] X. Zhu, Y. Ge, T. Wu et al., "Co-infection with respiratory pathogens among COVID-2019 cases," Virus Research, vol. 285, article 198005, 2020.

[49] Z. T. Li, Z. M. Chen, L. D. Chen et al., "Co-infection with SARS-CoV-2 and other respiratory pathogens in patients with COVID-19 in Guangzhou, China," Journal of Medical Virology, vol. 92, no. 11, pp. 2381-2383, 2020.

[50] M. T. T. Htar, M. Yerramalla, J. Moïsi, and D. Swerdlow, “The burden of respiratory syncytial virus in adults: a systematic 
review and meta-analysis," Epidemiology \& Infection, vol. 148, 2020.

[51] L. Lansbury, B. Lim, V. Baskaran, and W. S. Lim, "Co-infections in people with COVID-19: a systematic review and meta-analysis," Journal of Infection, vol. 81, no. 2, pp. 266275, 2020.

[52] B. Davis, A. N. Rothrock, S. Swetland, H. Andris, P. Davis, and S. G. Rothrock, "Viral and atypical respiratory co-infections in COVID-19: a systematic review and meta-analysis," Journal of the American College of Emergency Physicians Open, vol. 1, no. 4, pp. 533-548, 2020.

[53] Y. Si, Z. Zhao, R. Chen et al., "Epidemiological surveillance of common respiratory viruses in patients with suspected COVID-19 in Southwest China," BMC Infectious Diseases, vol. 20, no. 1, pp. 1-7, 2020.

[54] Z. Shen, Y. Xiao, L. Kang et al., "Genomic diversity of SARSCoV-2 in coronavirus disease, patients," Clinical Infectious Diseases, 2019.

[55] G. Härter, C. D. Spinner, J. Roider et al., "COVID-19 in people living with human immunodeficiency virus: a case series of 33 patients," Infection, vol. 48, pp. 681-686, 2020.

[56] J. M. Tesoriero, C. A. Swain, J. L. Pierce et al., "COVID-19 outcomes among persons living with or without diagnosed HIV infection in New York State," JAMA Network Open, vol. 4, no. 2, article e2037069, 2021.

[57] H. Mirzaei, W. McFarland, M. Karamouzian, and H. Sharifi, "COVID-19 among people living with HIV: a systematic review," AIDS and Behavior, vol. 25, no. 1, pp. 85-92, 2021.

[58] A. A. Butt and P. Yan, "Rates and characteristics of SARSCoV-2 infection in persons with hepatitis C virus infection," Liver International, vol. 41, no. 1, pp. 76-80, 2021.

[59] H. Mirzaie, M. Vahidi, M. Shokoohi et al., "Covid-19 among patients with hepatitis b or hepatitis c: A systematic review," Hepatitis Monthly, vol. 20, no. 11, pp. 1-2, 2020.

[60] W. Guo, M. Li, Y. Dong et al., "Diabetes is a risk factor for the progression and prognosis of COVID-19," Diabetes/metabolism research and reviews, vol. 36, no. 7, article e3319, 2020.

[61] S. A. Alqahtani and J. M. Schattenberg, "Liver injury in COVID-19: the current evidence," United European Gastroenterology Journal, vol. 8, no. 5, pp. 509-519, 2020.

[62] Y. J. Wong, M. Tan, Q. Zheng et al., "A systematic review and meta-analysis of the COVID-19 associated liver injury," Annals of Hepatology, vol. 19, no. 6, pp. 627-634, 2020.

[63] B. Cantan, C.-E. Luyt, and I. Martin-Loeches, "Serious infections in the ICU: evolving concepts in management and prevention: influenza infections and emergent viral infections in intensive care unit," in Seminars in respiratory and critical care medicine, Thieme Medical Publishers, 2019.

[64] S. Paolucci, I. Cassaniti, F. Novazzi et al., "EBV DNA increase in COVID-19 patients with impaired lymphocyte subpopulation count," International Journal of Infectious Diseases, vol. 104, pp. 315-319, 2021.

[65] A. Simonnet, I. Engelmann, A. S. Moreau et al., "High incidence of Epstein-Barr virus, cytomegalovirus, and humanherpes virus-6 reactivations in critically ill patients with COVID-19," Infectious Diseases Now, vol. 51, no. 3, pp. 296299, 2021.

[66] S. C. Kuo, H. H. Tsou, H. Y. Wu et al., "Nonpolio enterovirus activity during the COVID-19 pandemic, Taiwan, 2020," Emerging Infectious Diseases, vol. 27, no. 1, p. 306, 2021.
[67] B. W. Massey, K. Jayathilake, and H. Y. Meltzer, "Respiratory microbial co-infection with SARS-CoV-2," Frontiers in Microbiology, vol. 11, 2020.

[68] K. Holakouie-Naieni, E. Mostafavi, A. D. Boloorani, M. Mohebali, and R. Pakzad, "Spatial modeling of cutaneous leishmaniasis in Iran from 1983 to 2013," Acta Tropica, vol. 166, pp. 67-73, 2017.

[69] R. Pakzad, A. Dabbagh-Moghaddam, M. Mohebali, S. Safiri, and M. Barati, "Spatio-temporal analysis of cutaneous leishmaniasis using geographic information system among Iranian Army units and its comparison with the general population of Iran during 2005-2014," Journal of Parasitic Diseases, vol. 41, no. 4, pp. 1114-1122, 2017.

[70] R. Pakzad, I. Pakzad, S. Safiri et al., "Spatiotemporal analysis of brucellosis incidence in Iran from 2011 to 2014 using GIS," International Journal of Infectious Diseases, vol. 67, pp. 129136, 2018.

[71] N. Mahdavifar, R. Pakzad, M. Ghoncheh, I. Pakzad, A. Moudi, and H. Salehiniya, "Spatial analysis of breast cancer incidence in Iran," Asian Pacific Journal of Cancer Prevention, vol. 17, pp. 59-64, 2016.

[72] R. Pakzad, M. Ghoncheh, Z. Pournamdar et al., "Spatial analysis of skin cancer incidence in Iran," Asian Pacific Journal of Cancer Prevention, vol. 17, pp. 33-37, 2016.

[73] R. Pakzad, Y. Khani, I. Pakzad et al., "Spatial analysis of stomach cancer incidence in Iran," Asian Pacific Journal of Cancer Prevention, vol. 17, pp. 27-32, 2016.

[74] R. Pakzad, A. Moudi, Z. Pournamdar et al., "Spatial analysis of colorectal cancer in Iran," Asian Pacific Journal of Cancer Prevention, vol. 17, pp. 53-57, 2016.

[75] S. Soltani, A. Zakeri, M. Zandi et al., "The role of bacterial and fungal human respiratory microbiota in COVID-19 patients," Bio Med Research International, vol. 2021, pp. 1-23, 2021.

[76] P. Hosseini, S. Afzali, M. Karimi et al., "The coronavirus disease 2019 and effect on liver function," Reviews in Medical Microbiology, 2021. 